many places, there is a shoal at some distance from the lighthouse, or where a reef of rocks projects seawards from the shore, it sometimes becomes necessary to adopt means for keeping ressels clear of such dangers at night, as, for example, near Souter Point, where Mr. Douglass and Mr. J. T. Chance employed successfully the electric light for guarding a rock near the shore. What is wanted in such cases is to cover not only the danger itself but some area of the surrounding sea by a characteristic which is different from that of the main light.

If in front of a fixed light apparatus whose optical property is to parallelise the rays in the vertical plane while not interfering with their natural divergence in azimuth, there be placed an arrangement of straight horizontal shades or screens similar to the Venetian blinds which are used for house windows, the means will be supplied for easily producing different distinctions. The breadth of those blinds must be such as to subtend from the central flame the same angle as that over which the necessary distinction has to be shown at sea. By opening and shutting simultaneously and gradually the different leaves of the blind, there will be produced the same characteristics as those of an ordinary revolving or flashing light, according as the leaves are moved slowly or quickly and kept shut for a certain period, and these distinctions will be accompanied by the necessary gradual waxing and waning of the emergent rays. By simultaneously opening and shutting the leaves of the blind suddenly, and keeping them open so as to show a fixed light for a certain length of time, and then keeping them shut so as to produce darkness for a certain length of time, the effect of an intermittent light in which there is no waxing or waning of the rays will obviously be produced.

Should it be considered desirable to vary the appearance over the giren arc so as to show a gradually increasing length of light period when a vessel is approaching the danger, the maximum period when it is opposite to it, and a correspondingly shortening period as the vessel leaves it, a single straight opaque mask placed outside of the apparatus, and revolving horizontally and with uniform speed on a vertical spindle will produce the result. For while the periods of change will remain the same over the whole arc the duration of darkness will gradually increase as the danger is approached, and gradually decrease after the danger has been passed. And if this vertical shade be made to rotate at a slow and uniform speed it will produce the effect of a revolving light, and if at a quick speed it will produce the effect of a flashing light, with this difference that the flashes will recur ivith only an instantaneous interval of darkness, and in both cases there will be a gradual waxing and waning of the rays.

By these very simple and cheap expedients a fixed light illuminating the whole horizon (by means of a flame of he ordinary size in relation to the focal length) can easily be made to show accurately over any limited angle in azimuth the effects of the different distinctions referred to, and these combinations will therefore supply a deside. ratum which is often much wanted in coast illumination. In some experiments which were made all these characteristics were successfully produced by the two modes described.

Where no light is required in any part of the horizon but in one small arc only, as, for example, in illuminating the middle of a long narrow Sound, all the rays proceeding from the lamp should be spread equally over that arc. A fixed holophote with an opaque disk revolving horizontally in front on a vertical spindle will, if condensing prisms are placed between the disk and the holophote, produce either a revolving or flashing light according to the speed of its revolution, but without any intervening period of darkness. If colour distinction be required and a revolving disk of glass be substituted for the opaque mask the characteristic effect produced would be that of a revolving red and white light without any intervening dark period between the flashes, which would gradually dissolve into each other from red to white and then from white to red. Edinburgh, October 22 THOMAS STEVENSON

\section{A FEAT IN TRIANGULATION}

A NOTEWORTHY advance in geodesy has recently been accomplished by the junction of the network of measurements covering a large portion of the surface of Europe, with the African continent. The entire triangulation of Algeria was completed by French engineers some time since, and extended to the edge of the Sahara, in lat. $37^{\circ}$. M. Perrier, who had directed in a great measure the triangulation of Algeria, bas for the past eleven years been seeking the means of joining the network in that country with the perfect trigonometric system covering the surface of Spain, France, and England. The importance of such a junction is easily appreciated when we consider what notable changes in the accurate conception of the shape of the earth and of the length of meridians has been effected by measurements on a much smaller scale.

For such an undertaking the most careful and painstaking preparations were requisite. As the result of his reconnaissances between 1868 and 1872 , M. Perrier found that from ail the trigonometric points of the first order between Oran and the frontiers of Morocco, the loftier crests of the Sierra Nevada on the Spanish coast opposite, were visible in exceptionally clear weather. Arrangements were subsequently made with the Spanish Geographical Institute for the mutual and contemporaneous execution. of the proposed plan. A corps of Spanish officers, under the direction of the well-known General Ibanez, was detailed for this purpose, while the French Minister of War placed a division of officers from the Etat-Major under the command of $M$, Perrier. The leaders chose for stations in Algeria the summits of Mount Filhaoursen and Mount M'Sabiha, west of Oran, and in Spain the summits of Mount Tetica and Mount Mulhacen, the latter of which is the most elevated point in the kingdom. The directions and distances between these four points were computed as carefully as possible, and preparations were then made for the final and determinative observations. At the Algerian stations the nature of the country and its inhabitants necessitated the use of a numerous force of soldiery as well as of means of transport.

In order to insure the accuracy of the observations, which required the passage of signals over a distance of 270 kilometres, it was decided to make use of solar reflectors and powerful lenses. The efficacy of such apparatus for even greater distances had already been tested by M. Perrier; still for the measurements in question they appear to have utterly failed to answer the expectations based upon them, not a single solar signal being visible from any station. Fortunately, the success of the observations did not rest entirely upon this one system of signals. Preparations had likewise been made for the employment of the electric light, and on the summit of each mountain one of Gramme's electro-magnetic machines worked by engines of 6-horse power had been placed in position.

On August 20 last, all the stations were occupied, and the electric lights were displayed throughout each night. Then the patience of the observers was submitted to a lengthy proof. The mists rising from the Mediterranean totally prevented the exchange of signals, until after a delay of twenty days, one after another the electric lights became visible even to the naked eye. Perrier compared the intensity of the light on Tetica nearly 270 kilometres distant, to that of $a$ in Ursa Major, which rose near by. The observations were continued from September 9 to October I8, when this task for which such extensive preparations had been made, was completed in the most satisfactory manner. With its completion we come into 
possession of trigonometric measurements of the most exact nature, extending from lat. $61^{\circ}$ in the Shetland Islands, to lat. $34^{\circ}$ on the southern frontier of Algeria.

The extension of this network southward and eastward in Africa, desirable as it is for the elucidation of many nice points in geodesy, is unfortunately scarcely possible in the immediate future, and science must rest content with gaining a foothold in the great continent.

T. H. N.

\section{A NEW STANDARD OF LIGHT 1}

$\mathrm{I}$ the pamphlet before us we have a proposal for a new form of standard light, and the author has shown some considerable skill in drawing out his method of producing it. We cannot do better than quote his opening paragraph as showing the requisites of a standard that the author deems necessary. He says :-

"No exact measurement of any quantity, even with the most accurate and sensitive test measures available, can reasonably be expected unless the standard by which the unknown quantity is to be gauged is perfectly constant in itself; or if nature does not permit of such a desirable state of things, the causes to which the variation of the standard are due should be known, and in addition also, their quantitative effect on the standard, in order to be able to introduce a correction whenever accuracy of measurement should permit, and circumstances necessitate it."

The want of a standard of light has long been felt in physical researches, and the British Association has acknowledged the impossibility of obtaining scientific measures with the ordinary standards, and has appointed a committee to consider the question of fixing such a standard of white light, that a unit of light may be capable of accurate definition. It must not be forgotten that up to quite recent times the principal necessity for a standard at all has arisen through the introduction of gas into our dwellings and streets, and it has only been necessary to adopt one which should give the comparative illuminating powers of any variable qualities of gas. In fixing such a standard the points to be looked at were (I) that the standard should be capable of easy and exact reproduction; (2) that the colour of the light should be approximately the same; and (3) that in varying states of barometric pressure and temperature, proper corrections in the results of the comparisons should be feasible. It will be seen further on that a fourth desideratum should be introduced for scientific work. Perhaps on no subject has more attention been paid to small details than in the production of a standard candle, and as a result, when burnt under proper conditions, it gives fairly correct values of the illuminating power of gases.

In the record of Mr. Schwendler's experiments with the standard candle as against his new standard of light, we have some startling variations in the light of a standard candle, but we feel sure that, had the proper conditions been observed, there would never have occurred such a tremendous difference as 72 per cent. We are more convinced that ordinary precautions could not have beer rigidly observed when we find that some of the comparisons were made after the candle had been freshly lighted. In gas photometry it is well known that the standard candle should burn at least a quarter of an hour before it can be considered to have settled down to a steady light. The standard candle, however, is not a nice unit of light; and two years ago Mr. Vernon Harcourt introduced to the notice of the British Association a gas standard which seems to meet every requirement. By making a mixture in a small gasholder of one part of the most volatile spirit from American petroleum which distilled at $50^{\circ} \mathrm{C}$. with 600 of air,

In a New Standard of Light. By Louis Schwendler. From the Fournal of the Society of Bengal, vil. xlviii. Part ii., I879. or seven of the vapour with twenty of air, he produced a gas which, whilst almost insoluble in water, was permanent at all ordinary temperatures and pressures, and which was of a known composition and easy of manufacture. A jet of such a gas could be compared with the ordinary coal-gas, and any variations affecting the one would equally affect the other. The colours of the standard and coal-gas lights are also approximately the same. It seems that a standard of such a character meets the requirements for comparing the illuminating value of different coal-gases. Mr. Schwendler proposes to use the light radiated from platinum foil, when raised to incandescence by an electric current, as a new standard, and we agree that a solid instead of a gaseous body as the source of illumination is a step in the right direction. The standards made, however, appear to have been used for determining the illuminating value of the light produced by dynamo-electric machines under varying conditions of speed of armature and resistance in circuit, and it is in reference to this that we will first judge of its probable effectiveness, since for gas measurements the standards already existent suffice. Some dynamo-electric machines are advertised as generating the light of 50,000 candles, and we will suppose for the moment we are comparing such a light with Mr. Schwendler's standard.

Now it may be safely said that a standard candle, farther away than twenty feet from the photometer, would give too small a light to be practically of use as a standard, whilst if approaching the photometer within one foot the magnitude of the illuminating source would seriously affect any accurate results. In the first case the electric light would have to be about 4,500 feet away from the photometer and in the last about 220 feet. For ordinary photometric work even the least of these distances would be objectionable. The platinum standard employed by $\mathrm{Mr}$. Schwendler is only about ' 7 of a standard candle, and these distances would have to be increased nearly 20 per cent.

For practical measurements of this description a candlepower of fifty candles is a far preferable value, which it would be difficult to attain by the method proposed. In this case we have the distances reduced, and if the electric lamp is fixed at a distance of Ioo feet, we have the movable standard ranging between twenty feet and three to four feet, and the readings become easy and are not subject to be seriously affected by the magnitude of the illuminating source ; in fact, the errors of observation then become of larger magnitude than any error arising from this cause. Another point which we have to note is that as far as the colour of the light from the platinum standard is concerned, it possesses very little advantage over the ordinary gas or candle flame, and it would be impossible, or at all events incorrect, to give the illuminating value of a light such as of that produced by the electric arc in terms of the new standard; some recent experiments have demonstrated that the red light emitted by one square mile of the hollow crater in the positive carbon is equal to about the red light radiated by 40,000 standard candles, whilst the mean green light of the former is equal to the mean green light of about 135, coo of the latter, and until such a time as the relative physiological values of green and red light are accurately known it will be impossible to give any true estimate of the illuminating power of the electric light by ordinary photometric comparisons. Both in magnitude and colour, then, the proposed platinum standard of light seems to fail for measuring light produced by high temperatures.

We now turn to the details of the lamp itself. We have, firstly, a U-shaped piece of thin platinum foil cut out about $20 \mathrm{~mm}$. in total length, each limb of the $U$ being about $3 \mathrm{~mm}$. in breadth, the tops of which are clipped in thick metal clips. The usual arrangements are made for passing a current through this foil, the amount being registered by a galvanometer in circuit. A 